\title{
Shape of Polymer Chains Confined in Spheres
}

\author{
Mengbo Luo, Jianhua HuANG, ${ }^{*}$ and Jianmin XU \\ Department of Physics, Hangzhou University, Hangzhou 310028, \\ People's Republic of China \\ *Zhejiang Petrochemical Industry Development Co., Hangzhou 310007, \\ People's Republic of China
}

(Received February 16, 1998)

\begin{abstract}
A self-avoiding polymer chain confined in a sphere is generated by Monte Carlo technique on a simple cubic lattice. The dependence of shape of polymer chain on concentration $C$ of polymer solution is studied. The shape of polymer chain is denoted as $\left\langle L_{1}^{2}\right\rangle:\left\langle L_{2}^{2}\right\rangle:\left\langle L_{3}^{2}\right\rangle$, where $L_{1} \leq L_{2} \leq L_{3}$ are orthogonal components in the system of principal axes of gyration. At low concentration $(C \leq 0.001),\left\langle L_{2}^{2}\right\rangle \mid\left\langle L_{1}^{2}\right\rangle$ and $\left\langle L_{3}^{2}\right\rangle \mid\left\langle L_{1}^{2}\right\rangle$ remain approximately unchanged with the concentration. Then, with increase of the concentration, $\left\langle L_{2}^{2}\right\rangle /\left\langle L_{1}^{2}\right\rangle$ and $\left\langle L_{3}^{2}\right\rangle /\left\langle L_{1}^{2}\right\rangle$ decrease, and $\left\langle L_{3}^{2}\right\rangle /\left\langle L_{1}^{2}\right\rangle$ decreases more rapidly than $\left\langle L_{2}^{2}\right\rangle /\left\langle L_{1}^{2}\right\rangle$. Asymptotically, $\left\langle L_{2}^{2}\right\rangle /\left\langle L_{1}^{2}\right\rangle$ and $\left\langle L_{3}^{2}\right\rangle \mid\left\langle L_{1}^{2}\right\rangle$ become 1.0 at a concentration $C=1.0$.

KEY WORDS Shape / Monte Carlo Technique / Confined Polymer Chain /
\end{abstract}

As early as 1934, Kuhn pointed out that the most probable instantaneous shape of a polymer chain is ellipsoidal. ${ }^{1}$ Many studies showed that the limited values of $\left\langle L_{1}^{2}\right\rangle:\left\langle L_{2}^{2}\right\rangle:\left\langle L_{3}^{2}\right\rangle$ are about $1: 2.7: 12.0$ for many polymer chains in dilute solution. ${ }^{2-7}\left\langle L_{i}^{2}\right\rangle \mathrm{S}(i=1,2$, and 3 ), mean eigenvalues of the radius of gyration tensor $\boldsymbol{S}$, were introduced to study the shapes of flexible polymer chains by Šolc and Stockmayer. ${ }^{2,3}\left\langle L_{1}^{2}\right\rangle:\left\langle L_{2}^{2}\right\rangle:\left\langle L_{3}^{2}\right\rangle$ is taken as a measure of the shape of polymer chain. If the concentration of polymer solution varies or the polymer chain collapses, the instantaneous shape of the polymer chain may change.

In many situations one can regard the polymer chain as constrained in a sphere. For example, a polymer chain in a dilute or semidilute polymer solution can be treated as each chain or part of the chain being subjected to a sphere, ${ }^{8-10}$ the same as a collapsed polymer chain. ${ }^{11,12}$ Thus, the shape of the polymer chain may vary when the concentration of the polymer solution varies or the polymer chain goes from an extended swollen coil to a collapsed dense globule. One can simulate the increase of the concentration of the polymer solution or the collapse of the polymer chain by decreasing the dimensions of the shpere.

In the present work, the dependence of the shapes of polymer chain on the concentration of the polymer solution is studied. The model of polymer chain is a self-avoiding walk (SAW) on a simple cubic (SC) lattice. Polymer chains are simulated in spheres, which are taken to be completely permeable to solvent but whose walls are regarded as rigid constraints for the polymer chain. We find that $\left\langle L_{2}^{2}\right\rangle /\left\langle L_{1}^{2}\right\rangle$ and $\left\langle L_{3}^{2}\right\rangle /\left\langle L_{1}^{2}\right\rangle$ decrease with increase of the concentration of the polymer solution, especially when the concentration exceeds 0.001 , and $\left\langle L_{3}^{2}\right\rangle /\left\langle L_{1}^{2}\right\rangle$ decreases more rapidly than $\left\langle L_{2}^{2}\right\rangle /\left\langle L_{1}^{2}\right\rangle$. Asymptotically, they tend to become 1.0 at a concentration of 1.0 .

\section{CALCULATION METHOD}

The work considers a self-avoiding polymer chain of length $n$ consisting of $n$ segments and $n+1$ beads of unit mass, each bead locates on a simple cubic lattice. The polymer chain, which is confined in a sphere, is generated by the Monte Carlo technique. By the method, a chain is constructed by adding beads step-by-step. The first bead of a polymer chain is selected arbitrarily in the sphere. Then we scan the probable choices for the next bead by scanning the number $W_{k}(k=2,3, \cdots, n+1)$ of allowed lattice sites for the next bead from this lattice site. If $W_{k}=1$, we let the next bead occupy the only lattice site. If $W_{k}>1$, that is, there are more than one possible lattice site for the next bead to choose from, we let the computer generate randomly an integer among 1 to $W_{k}$, the lattice site for next bead is chosen according to the integer. Once there is no possible continuation in the procedure, i.e., $W_{k}=0$ for any $k$, then the construction is stopped, the uncompleted chain is abandoned and a new one is started.

When a polymer chain of length $n$ is successfully generated, the radius of gyration tensor $\boldsymbol{S}$ is calculated as

$$
\boldsymbol{S}=\frac{1}{n+1} \sum_{k=0}^{n} s_{k} \cdot S_{k}^{T}=\left(\begin{array}{ccc}
S_{x x} & S_{x y} & S_{x z} \\
S_{x y} & S_{y y} & S_{y z} \\
S_{x z} & S_{y z} & S_{z z}
\end{array}\right)
$$

where $s_{k}=\operatorname{col}\left(x_{k}, y_{k}, z_{k}\right)$ is the position vector of bead $k$ in a frame of reference with origin at the center of mass. $\boldsymbol{S}$ can be diagonalized by a judicious selected orthogonal matrix $Q$ to form a diagonal matrix

$$
\begin{aligned}
L & =Q^{-1} S Q \\
& =\operatorname{diag}\left(L_{1}^{2}, L_{2}^{2}, L_{3}^{2}\right)
\end{aligned}
$$

The operation known as diagonalization of a tensor is in fact an eigenvalue-eigenvector problem, which can be solved easily by computer. $L_{1}, L_{2}$ and $L_{3}\left(L_{1} \leq\right.$ $L_{2} \leq L_{3}$ ) are orthogonal components in the system of principal axes of gyration.

After generated a large number of chain samples, the statistical average $\left\langle L_{1}^{2}\right\rangle,\left\langle L_{2}^{2}\right\rangle$, and $\left\langle L_{3}^{2}\right\rangle$ are calculated as 


$$
\left\langle L_{i}^{2}\right\rangle=\frac{\sum_{j=1}^{N} L_{i}^{2} \cdot P_{j}}{\sum_{j=1}^{N} P_{j}} \quad(i=1,2,3)
$$

where $N$ is the number of chain samples and $P_{j}$ the Rosenbluth-Rosenbluth probability of chain $j .{ }^{13} P_{j}$ is named as

$$
P_{j}=\left(W_{2} / 6\right) \times \prod_{k=3}^{n+1}\left(W_{k} / 5\right)
$$

where $W_{k}$ is the number of allowed choices for the bead $k$ in the process of chain building. The maximum number of allowed choices for the second bead $(k=2)$ is six, while five for other beads $(k \geq 3)$.

\section{RESULTS AND DISCUSSION}

In this paper, the polymer chain lengths considered were $n=20,40,80$, and 120 with chain sample numbers $N=5000$ up to 20000 , increasing with chain length $n$. One reason that we did not consider much longer chain length is that, for an unconfined SAW chain on a simple lattice (which is similar to our model), the ratio $\left\langle L_{2}^{2}\right\rangle /\left\langle L_{1}^{2}\right\rangle$ and $\left\langle L_{3}^{2}\right\rangle /\left\langle L_{1}^{2}\right\rangle$ for chain length $n=120$ is almost the same for even longer chain according to the results of Bruns. ${ }^{14}$ Another reason is that it becomes difficult to build a long chain in spheres when the concentration reaches some value. For example, it was almost impossible to build a chain with length $n=240$ at concentration $C=0.5, \quad n=360$ at concentration $C=0.4$. The concentration of polymer solution, defined in the paper as the ratio of the lattice sites occupied by the polymer chain to the total lattice sites $V$ in the sphere

$$
C=\frac{n+1}{V}
$$

Polymer solution concentration $C$ varies from $10^{-5}$ to 0.6 in this work.

Figure 1 shows the dependence of $\left\langle L_{2}^{2}\right\rangle /\left\langle L_{1}^{2}\right\rangle$ and $\left\langle L_{3}^{2}\right\rangle /\left\langle L_{1}^{2}\right\rangle$ on the concentration for chain of length $n=120$. Dots and squares in Figure 1 are experimental data derived from the computer simulation, while the solid curves, as a guide to the eye, come from these experimental data using the least square method. The results for chain length $n=20,40$, and 80 are similar to that of $n=120$, thus we only show the results of $n=120$ here. Considering that $\left\langle L_{2}^{2}\right\rangle \mid\left\langle L_{1}^{2}\right\rangle$ and $\left\langle L_{3}^{2}\right\rangle \mid\left\langle L_{1}^{2}\right\rangle$ to be independent of chain length $n$ after reaching 120 for our chain model, we believe the results deduced from short chains should thus be valid for much longer chains. One finds from Figure 1 that $\left\langle L_{2}^{2}\right\rangle \mid\left\langle L_{1}^{2}\right\rangle$ and $\left\langle L_{3}^{2}\right\rangle /\left\langle L_{1}^{2}\right\rangle$ remain approximately constant while the concentration less than 0.001 . This indicates that the shape of polymer chain is almost independent of concentration when the chain is in the solution which concentration is less than 0.001

One could also find that both $\left\langle L_{2}^{2}\right\rangle \mid\left\langle L_{1}^{2}\right\rangle$ and $\left\langle L_{3}^{2}\right\rangle /\left\langle L_{1}^{2}\right\rangle$ decrease as the concentration increases when the concentration exceeds 0.001 , indicating that the deviation of shape of polymer chain from sphere

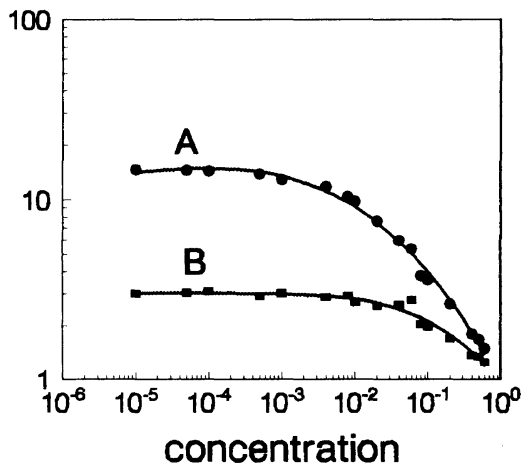

Figure 1. Log-log plots of $\left\langle L_{2}^{2}\right\rangle /\left\langle L_{1}^{2}\right\rangle$ and $\left\langle L_{3}^{2}\right\rangle /\left\langle L_{1}^{2}\right\rangle$ versus concentration for polymer chains confined in spheres. Curve $A$ and squares, $\left\langle L_{2}^{2}\right\rangle /\left\langle L_{1}^{2}\right\rangle$; curve $\mathrm{B}$ and dots, $\left\langle L_{3}^{2}\right\rangle /\left\langle L_{1}^{2}\right\rangle$.

decreases with increase of the concentration. Obviously, $\left\langle L_{3}^{2}\right\rangle /\left\langle L_{1}^{2}\right\rangle$ decreases more rapidly than $\left\langle L_{2}^{2}\right\rangle \mid\left\langle L_{1}^{2}\right\rangle$, revealing that $\left\langle L_{3}^{2}\right\rangle \mid\left\langle L_{1}^{2}\right\rangle$ is more susceptible to concentration. The probable reason is that $L_{3}$, the largest component of gyration, is the first to be restrained by the wall of sphere, $L_{2}$ is the second and $L_{1}$ is the last.

In the computer experiment, with an increase in concentration, it becomes more and more difficult to construct a polymer chain for a large proportion of conformations has to be abandoned before they have been constructed. ${ }^{10}$ Thus, simulation is not carried out for even larger concentration. Even so, it is not difficult to see that $\left\langle L_{2}^{2}\right\rangle /\left\langle L_{1}^{2}\right\rangle$ and $\left\langle L_{3}^{2}\right\rangle /\left\langle L_{1}^{2}\right\rangle$ tend to be 1.0 as the concentration approaches 1.0 .

In summary, the dependence of the shapes of polymer chains on the concentration of polymer solution is studied. Results show that, $\left\langle L_{2}^{2}\right\rangle \mid\left\langle L_{1}^{2}\right\rangle$ and $\left\langle L_{3}^{2}\right\rangle /\left\langle L_{1}^{2}\right\rangle$ decrease with the increase of concentration, $\left\langle L_{3}^{2}\right\rangle \mid\left\langle L_{1}^{2}\right\rangle$ decreases more rapidly than $\left\langle L_{2}^{2}\right\rangle /\left\langle L_{1}^{2}\right\rangle$, and they both tend to be 1.0 as the concentration approaches 1.0. From these results, one could image the variation of the shape of a polymer chain with the concentration, which goes from ellipsoid of large ellipticity to ellipsoid of small ellipticity with increase of the concentration. Results also show that $\left\langle L_{2}^{2}\right\rangle /\left\langle L_{1}^{2}\right\rangle$ and $\left\langle L_{3}^{2}\right\rangle \mid\left\langle L_{1}^{2}\right\rangle$ remain approximately unchanged with concentration when the concentration is less than 0.001 .

\section{REFERENCES}

1. W. Kuhn, Kolloid-Z, 68, 2 (1934).

2. K. Šlc and W. H. Stockmayer, J. Chem. Phys., 54, 2756 (1971).

3. K. Šolc, J. Chem. Phys., 55, 335 (1971).

4. J. Mazur, C. M. Guttman, and F. L. MacCrackin, Macromolecules, 66, 872 (1973)

5. D. Y. Yoon and P. J. Flory, J. Chem. Phys., 61, 5366 (1974).

6. D. N. Theodorou and U. W. Suter, Macromolecules, 18, 1206 (1985).

7. W. Bruns and W. Carl, Macromolecules, 24, 209 (1991).

9. P. G. de Gennes, "Scalling Concepts in Polymer Physics," Cornell Univ. Press, Ithaca, N.Y., 1979.

9. M. Aubouy, O. Guiselin, and E. Raphael, Macromolecules, 29, 7261 (1996).

10. M. Luo and J. Xu, Eur. Polym. J., 22, 1395 (1997).

11. K. Binder, "Application of the Monte Carlo Method in Statistical Physics," Springer, Berlin, 1984.

12. A. Buguin and F. B. Wyart, Macromolecules, 29, 4937 (1996).

13. M. N. Rosenbluth and A. W. Rosenbluth, J. Chem. Phys., 23, 356 (1955)

14. W. Bruns, Makromol. Chem., Theory and Simul., 1, 287 (1992). 